\title{
Link-level Performance of FM-UWB in the Interfered IEEE 802.15.6 Channel
}

\author{
Harri Viittala \\ Centre for Wireless Communications, \\ University of Oulu \\ P.O. Box 8000 \\ Oulu, Finland FI-90014 \\ harri.viittala@gmail.com
}

\author{
Matti Hämäläinen \\ Centre for Wireless Communications, \\ University of Oulu \\ P.O. Box 8000 \\ Oulu, Finland FI-90014 \\ matti.hamalainen@oulu.fi
}

\author{
Jari Iinatti \\ Centre for Wireless Communications, \\ University of Oulu \\ P.O. Box 8000 \\ Oulu, Finland FI-90014 \\ jari.iinatti@oulu.fi
}

\begin{abstract}
This paper provides simulation results of the frequency modulated ultra wideband (FM-UWB) system with channel coding in an interfered IEEE 802.15.6 channel. The paper discusses a FM-UWB receiver structure and presents the developed simulator model. The receiver applies a delay-line modulator followed by an amplitude modulation projection detection. A channel model used is the IEEE 802.15.6 channel model 3 for an on-body link. An interference is modeled as a colored Gaussian noise presenting an in-band IEEE 802.15.4 interferer. The simulation results show that the FM-UWB system can tolerate as low as $-6 \mathrm{~dB}$ signal-to-interference power ratios (SIR) in the studied scenarios.
\end{abstract}

\section{CCS CONCEPTS}

-Networks $\rightarrow$ Network simulations; Network performance analysis;

\section{KEYWORDS}

FM-UWB, Interference, Simulation, WBAN

\section{ACM Reference format:}

Harri Viittala, Matti Hämäläinen, and Jari Iinatti. 2017. Link-level Performance of FM-UWB in the Interfered IEEE 802.15.6 Channel . In Proceedings of International Conference on Body Area Networks, Dalian, People's Republic of China, September 2017 (BODYNETS'17), 4 pages.

DOI: $10.475 / 123 \_4$

\section{INTRODUCTION}

Typically, wireless body area networks (WBAN) refer to a wireless system of devices carried by a human or animal. The communication architecture can be divided into three communication tiers: intra-WBAN (personal), inter-WBAN (local) and beyond-WBAN (global) based on the proximity of occurred communication around a body. Consequentially, the intra-WBAN can be separated to inbody, on-body and off-body communications.

Wireless communications around or vicinity of a human body is very challenging. A radio signal propagates around a body via diffractions and reflections from extremities and surrounding environment. A direct signal propagating through a body is strongly attenuated. In addition, movement of extremities, variation in tissue permittivities and shape of a body involve. [3, 7, 13, 18]

The IEEE 802.15.6 standard defines narrowband, ultra wideband (UWB) and human body communication (HBC) physical (PHY) layers to be used in the intra-WBAN communication [9]. From these, UWB communications provides robustness in a dense multipath environment and a low power spectral density technology for WBAN [11]. The IEEE 802.15.6 standard defines two UWB PHY layers, i.e., impulse radio UWB (IR-UWB) and frequency modulated ultra wideband (FM-UWB) [9]. FM-UWB is a very simple UWB approach for low and medium data rates exploiting a double frequency modulation [6]. It applies a low-modulation index frequency shift keying (FSK) followed by a high modulation index frequency modulation (FM) to achieve a constant envelope UWB signal in the frequency domain. FM-UWB can be considered as an analog implementation of a spread-spectrum system, where a modulation index is equal to a spreading gain of a spread spectrum system. The performance of FM-UWB is studied, e.g., in [4-6, 1517]. As far as we know, the FM-UWB system is not investigated with Bose-Chadhuri-Hocquenghem (BCH) encoding in an interfered channel before. This paper contributes simulation results of the FM-UWB system with BCH encoding as defined in the IEEE 802.15.6 standard [9]. In addition, the system performance is tested against an in-band interference.

This paper is organized as follows. Section 2 introduces the concept of the FM-UWB PHY layer. The simulator model, channel model and interference are discussed in Section 3. It also describes the simulation parameters. The simulation results are given in Section 4, and the paper is concluded in Section 5.

\section{FREQUENCY MODULATED UWB}

FM-UWB is a simple low data rate UWB technique utilizing a double frequency modulation [6]. Basically, it constitutes an analog implementation of a spread spectrum system [2]. In FM-UWB, a data stream is modulated with a low-modulation index digital FSK followed by a high-modulation index FM. The modulation index is defined as $\beta=\Delta f / f_{m}$, where $\Delta f$ is the frequency deviation and $f_{m}$ is the frequency of a modulating signal. The modulation index can be chosen freely. A small index $(\beta<1)$ yields to a narrowband FM signal, whereas a large index $(\beta>>1)$ generates a UWB signal. A FM signal $s_{\mathrm{FM}}(t)$ modulated by a signal $m(t)$ having a frequency $f_{m}$ is expressed as [6]

$$
s_{\mathrm{FM}}(t)=A \sin \left(2 \pi f_{c} t-\beta \cos \left(2 \pi f_{m} t\right)+\varphi_{0}\right),
$$


where $f_{c}$ is the carrier frequency and $\varphi_{0}$ is the arbitrary but timeindependent constant phase. Due to wideband frequency modulation, the processing gain $P G_{\mathrm{FM}}$ at a receiver can be expressed as the ratio of the RF bandwidth $B_{\mathrm{RF}}$ and subcarrier bandwidth $B_{\mathrm{SUB}}$ as

$$
P G_{\mathrm{FM}}=10 \log _{10}\left(\frac{B_{\mathrm{RF}}}{B_{\mathrm{SUB}}}\right) \text {. }
$$

A received signal is demodulated with a delay-line FM demodulator followed by a FSK demodulator, as depicted in Figure 1.

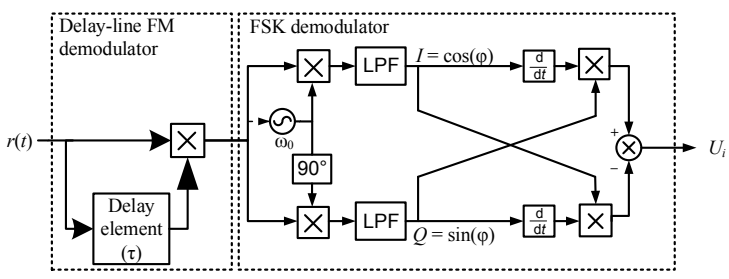

Figure 1: Block diagram of FM-UWB receiver

The received signal is multiplied with the $\tau=N_{d} /\left(4 f_{c}\right)$ delayed signal, where $N_{d}=1,3,5 \ldots$ [6] is selected so that it optimizes the useful bandwidth of the demodulator $B_{\text {DЕмод }}$ [6]. The useful bandwidth is the maximum frequency range where a demodulator transfer function is monotonic, defined as [6]

$$
B_{\mathrm{DEMOD}}=\frac{2}{N_{d}} f_{c} \text {. }
$$

The output voltage of the FM demodulator $V_{\mathrm{FM}, \text { out }}$ is given as [6]

$$
V_{\mathrm{FM}, \text { out }}(t)=(-1)^{\left(N_{d}+1\right) / 2} \frac{A^{2}}{2} \sin \left(O \frac{\pi}{2} \sin \left(\omega_{m} t\right)\right),
$$

where $O=N_{d}\left(\Delta f / f_{c}\right)$ is the FM demodulator overdrive and $\omega_{m}$ is the angular frequency of a modulating signal. The overdrive defines the ratio between a deviation of the FM signal and a useful bandwidth of the demodulator. From Equation (4), it can be seen that when the overdrive is less than 1 , the output voltage of the demodulator does not exploit the full available dynamic range. This occurs when a deviation of the FM signal is less than one half of the useful bandwidth of the modulator [6].

The output of the FM demodulator is followed by the FSK demodulator employing an amplitude modulation (AM) projection detection [10]. It converts an input to zero frequency and subsequently converts it to an AM wave with a differentiation stage and finally uses the AM-projection detection.

\section{SIMULATOR MODEL}

The software simulator implemented in Matlab was applied to study performance of the FM-UWB system. The simulator consists of a transmitter, receiver and channel. Random generated data bits are encoded by applying the $\mathrm{BCH}(63,51)$ code followed by FSK and FM demodulators. A transmitted signal $s(t)$ propagates through a multipath channel having an impulse response $h(t)$. An interference $i(t)$ and additive white Gaussian noise (AWGN) $n(t)$ are added to the signal yielding to a received signal $r(t)$

$$
r(t)=s(t) * h(t)+i(t)+n(t),
$$

where $*$ denotes the convolution operator.
The receiver performs demodulation and decoding. Received bits are compared to the data bits to have a bit error rate (BER) and frame error rate (FER). Figure 2 gives a block diagram representation of the simulator model.

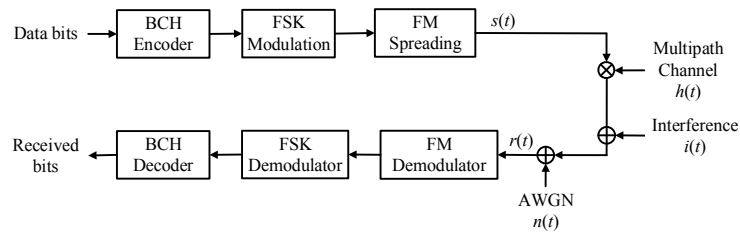

Figure 2: Block diagram of the FM-UWB simulator

\subsection{IEEE 802.15.6 Channel Model}

The simulator model uses the IEEE 802.15.6 channel model for a body-to-body link [18]. The model is based on the measurements carried out in a hospital room where the applied frequency band was from $3.0 \mathrm{GHz}$ to $11.0 \mathrm{GHz}$ [1]. A subject was laying down in a bed in a room of $5.0 \mathrm{~m}$ by $7.0 \mathrm{~m}$, a receive antenna was placed on the middle of a torso and a location of a transmit antenna varies from a head to an ankle. The distance between the antennas was from $176 \mathrm{~mm}$ to $984 \mathrm{~mm}$. The $S_{21}$-parameter was measured 10 times for each location.

A single cluster model where a path amplitude $a_{l}$ is modeled by an exponential decay $\Gamma$ with a Rician factor $\gamma_{0}$ was extracted as [18]

$$
10 \log _{10}\left|a_{l}\right|^{2}= \begin{cases}0, & l=0 \\ \gamma_{0}+10 \log _{10}\left(\exp \left(-\frac{t_{l}}{\Gamma}\right)\right)+S, & l \neq 0,\end{cases}
$$

where $S$ is the normal distribution with zero-mean and a standard deviation of $\sigma_{S}$. A path arrival time $t_{l}$ is modeled by the Poisson distribution [1]

$$
p\left(t_{l} \mid t_{l-1}\right)=\lambda \exp \left(-\lambda\left(t_{l}-t_{l-1}\right)\right),
$$

where $\lambda$ is the path arrival rate. The number of arrival paths $L$ are modeled by the Poisson distribution, given as[1]

$$
p(L)=\frac{\bar{L}^{L} \exp (\bar{L})}{L !},
$$

where $\bar{L}$ is the average number of $L . \phi_{l}$ is modeled by a uniform distribution over $[0,2 \pi)$. The parameter values for the channel model are tabulated in Table 1.

Table 1: Parameters for the IEEE 802.15.6 CM3

\begin{tabular}{lllll}
\hline$\Gamma[\mathrm{ns}]$ & $\gamma_{0}[\mathrm{~dB}]$ & $\sigma_{S}$ & $\lambda[1 / \mathrm{ns}]$ & $\bar{L}$ \\
\hline 59.7 & -4.60 & 5.02 & 0.541 & 38.1 \\
\hline
\end{tabular}

\subsection{Interference}

The simulator applies the colored Gaussian noise (CGN) noise to model an in-band interference, i.e., the white Gaussian noise process filtered by a bandpass filter. Using the band-limited characteristic 
of CGN, it is easy to implement specific interferences to the defined frequencies with the desired bandwidths. The interference generation procedure is presented in [14].

The bandpass filter uses the raised cosine waveform to have a baseband impulse response $h_{\mathrm{rc}}(t)$ having spectrum $H_{\mathrm{rc}}(f)$ as [12]

$$
H_{\mathrm{rc}}(f)= \begin{cases}T & 0 \leq|f|<\frac{1-\alpha}{2 T} \\ \frac{T}{2}\left(1+\cos \left(\frac{\pi T}{\alpha}\left(|f|-\frac{1-\alpha}{2 T}\right)\right)\right) & \frac{1-\alpha}{2 T} \leq|f| \leq \frac{1+\alpha}{2 T} \\ 0, & |f|>\frac{1+\alpha}{2 T}\end{cases}
$$

where $\alpha$ is the roll-off factor and $T$ is the symbol time related to the desired bandwidth $B W$. The filter is shifted to the desired center frequency $f_{c}$ as

$$
h_{\mathrm{cgn}}(f)=h_{\mathrm{rc}}(t) \cos \left(2 \pi f_{c} t+\varphi\right)
$$

An in-band interference $i(t)$ is generated by filtering the white Gaussian noise $n(t)$ with a filter $h_{\text {cgn }}$ having a frequency response $H_{\text {cgn }}(f)$ as illustrated in Figure 3. An interference power is adjusted according to a given signal-to-interference power ratio (SIR).

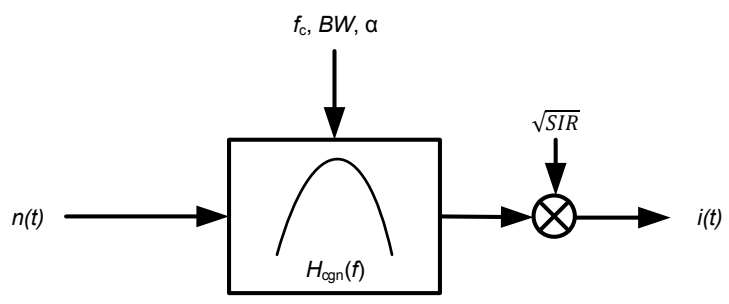

Figure 3: Generation of the interference

\subsection{Simulation Parameters}

The simulation parameters are given in Table 2. The first frequency band from the high band group was selected as defined in the IEEE 802.15.6 standard, i.e., the channel number 3 at the 6489.6 $\mathrm{MHz}$ center frequency and the bandwidth is $499.2 \mathrm{MHz}$ [9]. The IEEE 802.15.6 standard defines the uncoded bit rate of $250 \mathrm{kbps}$ for FM-UWB as a mandatory rate [9]. When using the $\mathrm{BCH}$ code $(63,51)$, the bit rate is $202.5 \mathrm{kbps}$. A modulating-carrier signal in FSK follows a triangular waveform. The selected parameters gives $N_{d}=27$ and $O=1.038$, which means that the FM demodulator is working almost an optimal range [6].

Table 2: Simulation Parameters

\begin{tabular}{ll}
\hline Parameter & Value \\
\hline Operating frequency band & 3 \\
Channel center frequency [MHz] & 6489.6 \\
Bandwidth [MHz] & 499.2 \\
Uncoded bit rate [kbps] & 250 \\
BCH coding & $(63,51)$ \\
Packet size, MPDU [octets] & 50 \\
FSK waveform & triangular \\
Modulation index, FSK & 1 \\
\hline
\end{tabular}

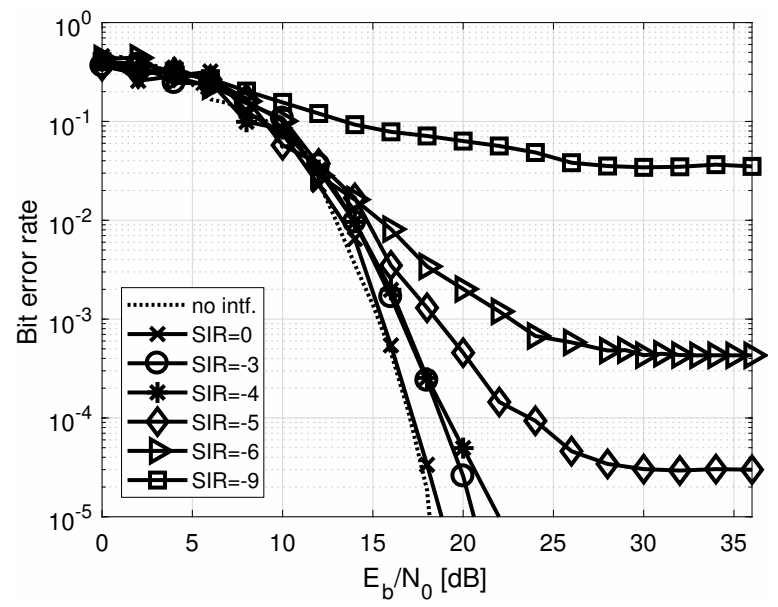

Figure 4: BER of uncoded FM-UWB

The applied channel model is the IEEE 802.15.6 channel model 3 as introduced in Section 3.1. An in-band interference is considered to be an IEEE 802.15.4 UWB system with the same bandwidth and center frequency as a victim IEEE 802.15.6 FM-UWB system, i.e., 6489.6 MHz and 499.2 MHz, respectively [8]. The roll-off factor for the raised cosine filter is 0.5 .

\section{SIMULATION RESULTS}

This section gives the simulation results of the uncoded and coded FM-UWB system. The performance of the system was simulated in the IEEE 802.15.6 channel model 3 without or with an IEEE 802.15.4 in-band interference. The channel model 3 models a body-to-body communication link.

Figure 4 and Figure 5 represent the uncoded BER and FER performances, respectively. The dotted line without markers is the reference performance without interference. A SIR value is reduced from $0 \mathrm{~dB}$ to $-9 \mathrm{~dB}$, i.e., the interference power is increased. As it can be seen from the results, the performance saturates when SIR is equal or less than $-5 \mathrm{~dB}$. Otherwise, a reliable communication link can be established in the IEEE 802.15.6 channel model 3 even when an interferer is present.

The $\mathrm{BCH}$ code improves the system performance, and hence, it tolerates higher SIR level as showed in Figures 6 and 7. The coding improves the system performance approximately $3 \mathrm{~dB}$ at the BER level of $10^{-5}$ without interference. The higher interference power can be tolerated with encoding, i.e., SIR of $-6 \mathrm{~dB}$. FM-UWB takes advantage of a large receiver processing gain to mitigate an in-band interference. After the FM demodulator, the part of the demodulated interference signal falls inside the subcarrier bandwidth. This explains a good performance with an in-band interference.

\section{CONCLUSION}

FM-UWB is a UWB PHY layer included in the IEEE 802.15.6 standard [9]. It is a simple technique where a double frequency modulation is applied to form an UWB waveform.

This paper presented the simulation results of the FM-UWB system with and without the $\mathrm{BCH}$ encoding in the interfered IEEE 


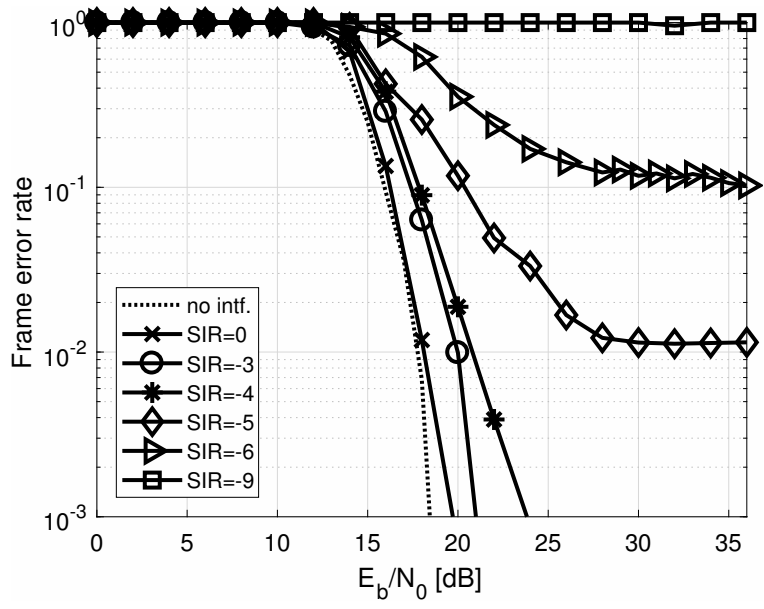

Figure 5: FER of uncoded FM-UWB

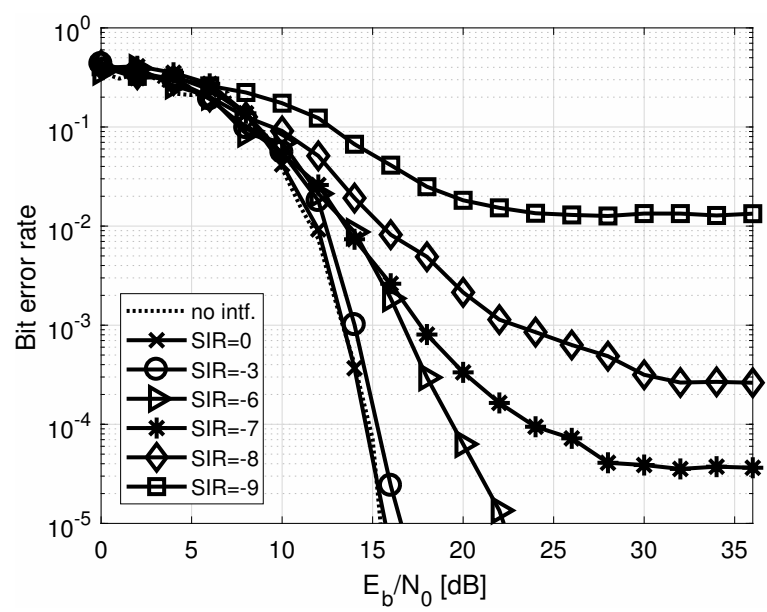

Figure 6: BER of coded FM-UWB

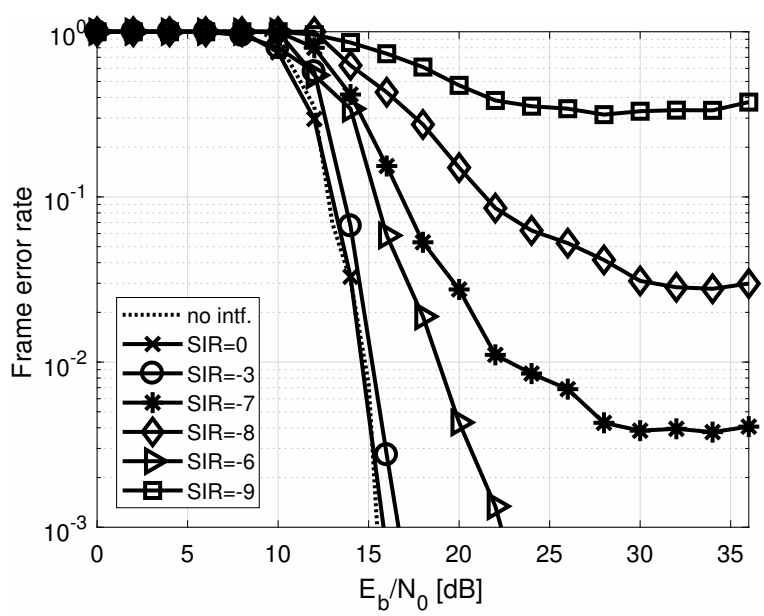

Figure 7: FER of coded FM-UWB
802.15.6 channel model 3. The interference was modeled as CGN and it was intended to simulate an in-band IEEE 802.15.4 system. As the results showed, the FM-UWB system performs well in moderate interference environments, i.e., SIR is less than $-6 \mathrm{~dB}$.

Future work could include modeling of the IEEE 802.15.4 interference system in detail.

\section{ACKNOWLEDGEMENT}

Responsible author would like to thank Ulla Tuominen and Seppo Säynäjäkangas Foundations for financing the work with personal grants.

\section{REFERENCES}

[1] T. Aoyagi, J. ichi Takada, K. Takizawa, H. Sawada, N. Katayama, K. Y. Yazdandoost, T. Kobayashi, H.-B. Li, and R. Kohno. 2008. Channel Models for WBANs - NICT. Technical Report IEEE P802.15-08-0416-04-0006. IEEE P802.15 Wireless Personal Area Networks.

[2] J. Farserotu, J. Gerrits, J. Rousselot, G. van Veenendaal, M. Lobeira, and J. Long. 2009. CSEM FM-UWB Proposal. Technical Report IEEE P802.15-09-0721-00-0006. IEEE P802.15 Wireless Personal Area Networks.

[3] A. Fort, C. Desset, P. D. Doncker, P. Wambacq, and L. V. Biesen. 2006. An Ultra-Wideband Body Area Propagation Channel Model: From Statistics to Implementation. IEEE Transactions on Microwave Theory and Techniques 54, 4 (June 2006), 1820-1826. DOI : http://dx.doi.org/10.1109/TMTT.2006.872066

[4] J. F. M. Gerrits, J. R. Farserotu, and J. R. Long. 2008. Low-Complexity Ultra-WideBand Communications. IEEE Transactions on Circuits and Systems II: Express Briefs 55, 4 (April 2008), 329-333.

[5] J. F. M. Gerrits, J. R. Farserotu, and J. R. Long. 2011. Robustness and Interference Mitigation for FM-UWB BAN Radio. In 2011 th International Symposium on Medical Information and Communication Technology. 98-102. DOI : http://dx.doi. org/10.1109/ISMICT.2011.5759805

[6] J. F. M. Gerrits, M. H. L. Kouwenhoven, P. R. van der Meer, J. R. Farserotu, and J. R. Long. 2005. Principles and Limitations of Ultra-wideband FM Communications Systems. EURASIP fournal on Applied Signal Processing 2005 (Jan 2005), 382-396. DOI : http://dx.doi.org/10.1155/ASP.2005.382

[7] M. Hämäläinen, A. Taparugssanagorn, and J. Iinatti. 2011. On the WBAN Radio Channel Modelling for Medical Applications. In Proceedings of the 5th European Conference on Antennas and Propagation (EUCAP). 2967-2971.

[8] IEEE. 2011. IEEE Standard for Local and Metropolitan Area Networks-part 15.4: Low-rate Wireless Personal Area Networks (LR-WPANs). IEEE Std 802.15.4-2011 (Revision of IEEE Std 802.15.4-2006) (Sept 2011), 1-314. DOI : http://dx.doi.org/10. 1109/IEEESTD.2011.6012487

[9] IEEE. 2012. IEEE Standard for Local and Metropolitan Area Networks - Part 15.6: Wireless Body Area Networks. IEEE Std 802.15.6-2012 (Feb 2012), 1-271. DOI : http://dx.doi.org/10.1109/IEEESTD.2012.6161600

[10] M. H. Kouwenhoven. 1998. High-Performance Frequency-Modulation Systems. Ph.D. Dissertation. Technische Universiteit Delft, the Netherlands.

[11] I. Oppermann, M. Hämäläinen, and J. Iinatti (Eds.). 2004. UWB: Theory and Applications. John Wiley \&Sons Inc.

[12] J. Proakis. 2001. Digital Communications. McGraw-Hill.

[13] A. Taparugssanagorn, C. P. Raez, A. Isola, R. Tesi, M. Hämäläinen, and J. Iinatti. 2010. UWB Channel Modelling for Wireless Body Area Networks in A Hospital. International fournal of Ultra Wideband Communications and Systems 1, 4 (2010), 226-236. DOI: http://dx.doi.org/10.1504/IJUWBCS.2010.034304

[14] R. Tesi. 2004. Ultra Wideband System Performance in the Presence of Interference. (2004). University of Oulu, Department of Electrical and Information Engineering, Licentiate Thesis.

[15] H. Viittala, M. Hämäläinen, and J. Iinatti. 2008. Suitability Study of DS-UWB and UWB-FM for Medical Applications. In The 11th International Symposium on Wireless Personal Multimedia Communications. 1-5.

[16] H. Viittala, M. Hämäläinen, and J. Iinatti. 2010. Impact Of Difference In WBAN Channel Models On UWB System Performance. In 2010 IEEE 11th International Symposium on Spread Spectrum Techniques and Applications. 175-180. DOI: http://dx.doi.org/10.1109/ISSSTA.2010.5651033

[17] H. Viittala, B. N. Nahar, M. Hämäläinen, and J. Iinatti. 2010. Medical Applications Adapting Ultra Wideband: A System Study. International fournal of Ultra Wideband Communications and Systems 1, 4 (2010), 237-247. DOI: http://dx.doi.org/10.1504/IJUWBCS.2010.034305

[18] K. Y. Yazdandoost and K. Sayrafian-Pour. 2009. Channel Model for Body Area Network (BAN). Technical Report IEEE P802.15-08-0780-09-0006. IEEE P802.15 Wireless Personal Area Networks. 